\title{
Ciclos de Vida de Pesquisa com base na Ciência Aberta
}

\author{
Larissa Mariany Freiberger Pereira ${ }^{1}$, Roberto Carlos dos Santos Pacheco ${ }^{1}$
}

${ }^{1}$ Programa de Pós-graduação em Engenharia e Gestão do Conhecimento - Universidade Federal de Santa Catarina (UFSC) - Florianópolis - SC - Brasil

freiberger.lm@gmail.com, rpacheco@egc.ufsc.br

\begin{abstract}
This paper sought to analyze, through a narrative review, the research lifecycle models presented in the literature and universities developed from the Open Science worldview. It was found that there are few research lifecycle models built from this perspective and, still, very limited, since they do not encompass distinct pillars on which the construct Open Science is understood. Finally, important aspects of Open Science were discussed that impact, in some way, the research life cycle. It was also observed the need for the development of research life cycles that embrace the fundamentals of Open Science so that researches are adherent to this movement.
\end{abstract}

Resumo. O presente artigo buscou analisar, por meio de uma revisão narrativa, os modelos de ciclo de vida de pesquisa apresentados na literatura e universidades desenvolvidos a partir da visão de mundo da Ciência Aberta. Verificou-se que existem poucos modelos de ciclo de vida de pesquisa construidos a partir desta perspectiva e, ainda, muito limitados, uma vez que não abarcam pilares distintos sobre os quais o constructo Ciência Aberta é compreendido. Observou-se, ainda, a necessidade do desenvolvimento de ciclos de vida de pesquisa que abracem os fundamentos da Ciência Aberta para que as pesquisam sejam aderentes a este movimento.

\section{Introdução}

Segundo a Rede Nacional de Bibliotecas de Medicina ${ }^{1}$ (National Network of Libraries of Medicine - NNLM), "ciclo de vida de pesquisa" ou research lifecycle é o termo usado para descrever o processo cíclico executado por pesquisadores para desenvolver uma pesquisa, desde a concepção de ideias até a implementação da estratégia de pesquisa e a disseminação do conhecimento, voltando à concepção de ideias, uma vez que, ao fim de uma pesquisa, novos insights deverão surgir e darão vida a novas pesquisas (Gessner et al. 2017; Toli et al. 2018). Para Ragon (2019, p. 384), "os modelos de ciclo de vida de pesquisa surgiram na tentativa de definir o fluxo de trabalho da pesquisa, desde a identificação do problema até a mensuração do impacto das descobertas publicadas".

Os modelos de ciclo de vida de pesquisa são inúmeros e podem ser encontrados na literatura ou podem também ser propostos por bibliotecas. Nestes modelos estão identificadas e descritas as etapas que compõem este processo (UCF Libraries $^{2}$ ), bem

\footnotetext{
${ }^{1}$ Programa nacional coordenado pela Biblioteca Nacional de Medicina (National Library of Medicine NLM) dos Estados Unidos.

${ }^{2}$ Disponível em: <https://library.ucf.edu/about/departments/scholarly-communication/overview-researchlifecycle/>. Acesso em: 18 set. 2019.
} 
como as atividades inerentes a cada uma das etapas (Library of Simon Fraser University $^{3}$ ). Não existe um modelo pré-definido aplicável aos mais diversos contextos. Ao contrário, o que se vê, é uma concepção genérica do que venha a ser um ciclo de vida de pesquisa e, a partir desta, os modelos são desenvolvidos e adaptados às necessidades específicas de um grupo dos pesquisadores ou da comunidade científica em que este deverá ser utilizado. A Lifecycle Initiative ${ }^{4}$ afirma que as abordagens baseadas em ciclo de vida, de modo geral, auxiliam no processo de tomada de decisão, uma vez que todos os envolvidos em todo o ciclo de vida, de seu início a seu fim, possuem responsabilidades e um papel a ser desempenhado, levando em consideração todos os impactos relevantes no contexto em que se insere.

No contexto científico, o desenvolvimento e uso de modelos de ciclo de vida de pesquisa pode favorecer o avanço da transparência em dois níveis: (1) em relação ao processo científico como um todo e (2) em relação às contribuições de cada participante engajado no processo de pesquisa. Este fato é consideravelmente relevante quando se pensa nas mudanças que acontecem atualmente no contexto científico, onde a ciência, com suas raízes fundadas em um modelo tradicional, começa migrar para um modelo aberto: a Ciência Aberta. O movimento Ciência Aberta engloba diversos conceitos, como o acesso aberto às publicações, compartilhamento de dados científicos, colaboração a partir do uso das plataformas de Tecnologias de Informação e Comunicação (TICs), open source, open notebooks, ciência cidadã, entre outros. A Ciência Aberta representa uma abordagem alternativa ao modelo tradicional de ciência. Esta abordagem abrange todo o ciclo de pesquisa ao ponto de gerar novas reflexões inclusive sobre o modo que os pesquisadores são avaliados e recompensados (Funamori, 2017).

Na literatura não existe uma definição universal acerca do constructo "Ciência Aberta". Fecher e Friesike (2011, p. 43) caracterizam a Ciência Aberta como um umbrela term, no sentido de que trata-se de um termo abrangente, "que evoca entendimentos bastante diferentes, dependendo do ponto de vista de seus respectivos advogados". Nesse sentido, após vasta revisão na literatura, os autores chegaram à conclusão de que o constructo "Ciência Aberta" pode ser abordado a partir de cinco visões de mundo distintas e apresentam cinco escolas de pensamento que estudam o tema a partir de perspectivas diferentes, a saber: (1) infraestrutura: ferramentas e plataformas abertas; (2) publicidade: a ciência é pública e os cidadãos devem estar envolvidos no processo científico; (3) mensuração: são necessárias métricas alternativas para mensuração de impacto da pesquisa aberta; (4) democracia: o conhecimento é público e deve estar acessível a todos; e (5) pragmatismo: a produção de conhecimento pode ser mais eficiente se os cientistas trabalharem juntos. De fato, a Ciência Aberta não pode ser vista de maneira isolada, mas é necessário que sejam analisados os diferentes pilares que sustentam o constructo, como questões de infraestrutura, acesso aberto, ciência cidadã, métricas para mensuração de impacto, transparência, entre outros.

Para Picarra (2016), a Ciência Aberta abrange diversos conceitos, ferramentas, plataformas e mídias. Conceitos como acesso aberto, metodologias abertas, dados abertos de pesquisa, educação aberta, avaliação aberta, altmetrics, ciência cidadã, open source,

\footnotetext{
${ }^{3}$ Disponível em: <https://www.lib.sfu.ca/about/branches-depts/rc/research/research-lifecycle>. Acesso em: 18 set. 2019.

${ }^{4}$ Parceria público-privada, com diversos stakeholders, engajada em permitir o uso global de conhecimento credível do ciclo de vida por tomadores de decisão nos âmbitos público e privado.
} 
entre outros. Ferramentas como softwares de código aberto, softwares de gerenciamento de bibliografia, open notebooks, open annotations, bibliografias abertas. Diferentes plataformas, especialmente aquelas dedicadas a crowdsourcing, pesquisa colaborativa, compartilhamento de pesquisa e engajamento entre pesquisadores e sociedade. Mídias online, tais como blogs, Twitter, Facebook, LinkedIn, entre outros.

Diversos modelos de ciclo de vida de pesquisa podem ser encontrados na literatura ou também em domínios de bibliotecas de universidades. Uma parte desses modelos são desenvolvidos tendo como objetivo promover a Ciência Aberta. O presente artigo tem como objetivo analisar as especificidades de modelos de ciclo de vida de pesquisa baseados na Ciência Aberta encontrados na literatura. Nesse sentido, cabe ressaltar que foi encontrado na literatura até o presente momento um trabalho uma revisão de literatura intitulado $A$ critical analysis of lifecycle models of the research process and research data management, de Cox e Tam (2018). Os autores fazem uma análise crítica de nove modelos, entre modelos de ciclo de vida de pesquisa e modelos de ciclo de vida de gerenciamento de dados de pesquisa, evidenciando pontos fortes e fracos desses modelos. Cox e Tam (2018) fazem uma análise ampla, envolvendo modelos de ciclo de gerenciamento de dados de pesquisa, que não é o objetivo deste artigo. Buscamos, então, aprofundar a análise de modelos que tratem especificamente de ciclos de vida de pesquisa.

\section{Procedimentos Metodológicos}

O presente artigo foi construído a partir de uma revisão narrativa da literatura. A revisão narrativa tem como objetivo não a apresentação de novos dados, mas a sintetização dos dados já existentes. Na revisão narrativa da literatura é possível trabalhar com um escopo amplo da pesquisa e esta característica, em especial, foi fundamental para a escolha desse tipo de revisão na condução da pesquisa (FESSARI, 2015).

Inicialmente foi realizada busca na base de dados Scopus por artigos ou revisões publicados em periódicos ou conferências com os termos research cycle ou research lifecycle e open science em seus títulos, palavras-chave ou resumos publicados até o dia 28 de março de 2020. A busca retornou 10 documentos. Posteriormente foram analisados títulos e resumos dos documentos e foi verificado que apenas dois deles apresentavam alguma proposta de ciclo de vida de pesquisa. Esses dois documentos foram analisados e serão apresentados na próxima seção. Dado a escassez de ciclos de vida de pesquisa baseados em Ciência Aberta na literatura, foram incluídos também na pesquisa ciclos de vida de pesquisa desenvolvidos por universidades. De fato, a busca na literatura não retorna resultados abrangentes quando se trata de ciclos de vida de pesquisa. Cox e Tam (2018, p. 3), sobre a busca na literatura, afirmam que "inevitavelmente, essa coleção não foi abrangente". Para dar solidez à pesquisa, os autores optaram por incluir modelos desenvolvidos por profissionais que estudam o tema e optaram por publicar seus estudos em meios alternativos, em relação aos periódicos revisados por pares. Semelhante aos autores, para dar solidez e tornar a pesquisa mais robusta, optamos por incluir na análise os modelos de ciclo de vida de pesquisa desenvolvidos por universidades e aplicados nestes mesmos ambientes. 


\section{Modelos de Ciclo de Vida de Pesquisa da Literatura baseados em Ciência Aberta}

Os dois artigos encontrados na literatura que propõem ciclos de vida de pesquisa baseados na Ciência Aberta são de Lyon (2016) e de Förstner et al. (2011).

Os autores têm um entendimento semelhante acerca dos fundamentos da Ciência Aberta. Para Lyon (2016), o constructo se fundamenta na participação, no acesso e na transparência. A participação abrange o grau de colaboração na pesquisa, podendo variar de um único pesquisador, passando por equipes de pesquisadores até a ciência cidadã, onde cidadãos comuns e outros setores da sociedade estão inseridos como pesquisadores no processo de pesquisa. $\mathrm{O}$ acesso aborda a capacidade de livremente localizar e recuperar artigos, desde aqueles disponibilizados em fontes fechadas como periódicos de assinatura revisados por pares até aqueles disponibilizados em repositórios institucionais de acesso aberto. Em relação à transparência, Lyon (2016) a percebe como o resultado de uma série de práticas e comportamentos dos stakeholders da pesquisa que culminam na viabilização da reprodutibilidade da pesquisa. Lyon (2016, p. 154) propõe um ciclo de vida de pesquisa analisando "oportunidades para possíveis intervenções de transparência" e as situa no ciclo de vida de pesquisa.

Förstner et al. (2011) ressaltam a importância do acesso aos dados gerados em todo o processo de pesquisa; da transparência em relação ao processo de pesquisa como um todo, bem como em relação à participação e contribuição de cada ator; e da colaboração que deve ser apoiada por Ambientes Virtuais de Pesquisa (Virtual Research Environments ou VREs). A tecnologia desempenha papel crucial ao longo de todo o ciclo de vida de pesquisa proposto por Förstner et al. (2011), seja por meio do uso dos VREs para promover a colaboração ou por meio da automação dos processos de coleta e análise de dados, objetivando a transparência e reprodutibilidade da pesquisa, bem como a disponibilização dos dados produzidos em tempo real.

Tabela 1. Tópicos de Análise dos artigos de Lyon (2016) e Förstner et al. (2011)

\begin{tabular}{|c|c|c|}
\hline & Lyon (2016) & Förstner et al. (2011) \\
\hline Fundamentos & Participação, Acesso e Transparência & Colaboração, Acesso e Transparência \\
\hline Foco & Transparência & Tecnologia \\
\hline Fases & $\begin{array}{l}\text { 1. Design } \\
\text { 2. Planejamento } \\
\text { 3. Coleta, Busca e Aquisição de Dados } \\
\text { 4. Processamento, Visualização e Análise } \\
\text { de Dados } \\
\text { 5. Armazenamento } \\
\text { 6. Preparo } \\
\text { 7. Publicação, Preservação e Arquivamento } \\
\text { 8. Localização }\end{array}$ & $\begin{array}{l}\text { 1. Concepção e Planejamento } \\
\text { 2. Experimento e Geração de Dados } \\
\text { 3. Lançamento de Dados } \\
\text { 4. Análise de Dados } \\
\text { 5. Geração de Conhecimento } \\
\text { 6. Publicação Final }\end{array}$ \\
\hline $\begin{array}{l}\text { Representação } \\
\text { Gráfica }\end{array}$ & ( & Não \\
\hline $\begin{array}{l}\text { Objetivo do } \\
\text { Artigo }\end{array}$ & $\begin{array}{c}\text { Explorar o conceito de transparência no } \\
\text { âmbito da pesquisa científica }\end{array}$ & $\begin{array}{c}\text { Estudar o ciclo de vida de pesquisa em } \\
\text { VREs }\end{array}$ \\
\hline Aplicabilidade & Geral & Engenharias \\
\hline
\end{tabular}

Apesar de perceberem diferentes fundamentos no que tange à Ciência Aberta, os dois ciclos de vida de pesquisa propostos são desenvolvidos com base em um aspecto específico. Lyon (2016) desenvolve todo o ciclo de vida de pesquisa e o próprio artigo a partir do conceito de transparência. Já Förstner et al. (2011) abordam o impacto do uso 
da tecnologia, em especial dos VREs, em cada uma das fases do ciclo de vida de pesquisa. A Tabela 1 sumariza alguns tópicos de análise relevantes das propostas de Lyon (2016) e de Förstner et al. (2011).

Nenhum dos artigos definem satisfatoriamente cada uma das fases que compõem os respectivos ciclos de vida de pesquisa e isto faz com que falte robustez às propostas, uma vez que não existe uma orientação concisa sobre quais as atividades e tarefas que devem ser desempenhadas em cada uma das fases.

\section{Modelos de Ciclo de Vida de Pesquisa de Universidades baseados em Ciência Aberta}

Os Modelos de Ciclo de Vida de Pesquisa de Universidades são institucionais, isto é, são desenvolvidos pela própria instituição e adaptados às necessidades e realidades específicas de cada contexto institucional. Em geral, estes modelos apresentam as fases que compõem o ciclo de vida de pesquisa, bem como os serviços ofertados belas bibliotecas de suporte à pesquisa em cada uma dessas etapas. Embora apresentem fases diversas, o modelo proposto possui essas duas características. Foram encontrados dez Ciclos de Vida de Pesquisa propostos pelas seguintes universidades: Simon Fraser University, Monash University, McMaster University, Victoria University, Bournemouth University, Western Sydney University, University of Dayton, University of York, The University of Arizona e University of Central Florida. A maior parte das universidades representam graficamente o ciclo de vida de pesquisa, exceto The University of Arizona, Victoria University e Simon Frase University. Em geral, as universidades apresentam orientações sobre três aspectos fundamentais da Ciência Aberta: colaboração, acesso aberto e dados abertos.

No que tange à colaboração, as orientações são as mais diversas: (1) uso de mídias sociais para viabilizar a colaboração na pesquisa, (2) incentivo à colaboração com outros setores da sociedade; (3) incentivo à colaboração com pesquisadores de outras áreas de pesquisa, (4) apresentação de listas com potenciais colaboradores, (5) plataformas institucionais de perfil de pesquisadores para promover o networking, entre outros. Sobre o acesso aberto, as orientações, salvo exceções, giram em torno do incentivo à publicação de artigos nas vias verde (repositórios abertos) e dourada (periódicos de acesso aberto). Algumas universidades também possuem um fundo de financiamento para autores que optem por publicar seus trabalhos em periódicos abertos e precisam dispor de taxas de publicação.

As orientações sobre dados abertos são mais restritas e apenas quatro universidades tratam deste tema em seus ciclos de vida de pesquisa: Simon Fraser University, Monash University, University of York e University of Central Florida. Simon Fraser University apresenta uma lista de repositórios abertos e diversas disciplinas para que os pesquisadores possam publicar seus dados. Monash University orienta quanto à publicação de dados abertos de pesquisa, bem como quanto à citação de dados abertos utilizados por seus pesquisadores. University of York cita a possibilidade de publicação de dados abertos no seu ciclo de vida de pesquisa. Por fim, University of Central Florida orienta quanto ao uso de metadados, tesauros e taxonomias para auxiliar na recuperação dos dados produzidos ao longo do processo de pesquisa. 
Os ciclos de vida de pesquisa das universidades são normalmente desenvolvidos pelas bibliotecas destas instituições e têm como foco conectar os pesquisadores aos serviços de apoio ao pesquisador fornecidos pelas universidades. São ciclos de vida de pesquisa institucionais, adaptados às realidades específicas de cada contexto e são fundamentais para a institucionalização da pesquisa nas universidades. As universidades definem cada uma das fases de seus ciclos de vida de pesquisa e abordam as principais atividades que estão inseridas em cada uma dessas fases.

Consideramos importante ressaltar a proposta da University of Central Florida (UCF), visto que ele tem uma peculiaridade: trata-se de um ciclo de vida de pesquisa proposto por uma universidade, mas que é amplamente citado na literatura. A UCF propõe um modelo de ciclo de vida de pesquisa descritivo, composto por quatro subciclos (Deng \& Dotson, 2015) e em cada um deles são informados os serviços e ferramentas disponibilizados pela biblioteca da universidade, bem como pelo setor de pesquisa da universidade (The Office of Research and Commercialization) para apoiar os pesquisadores.

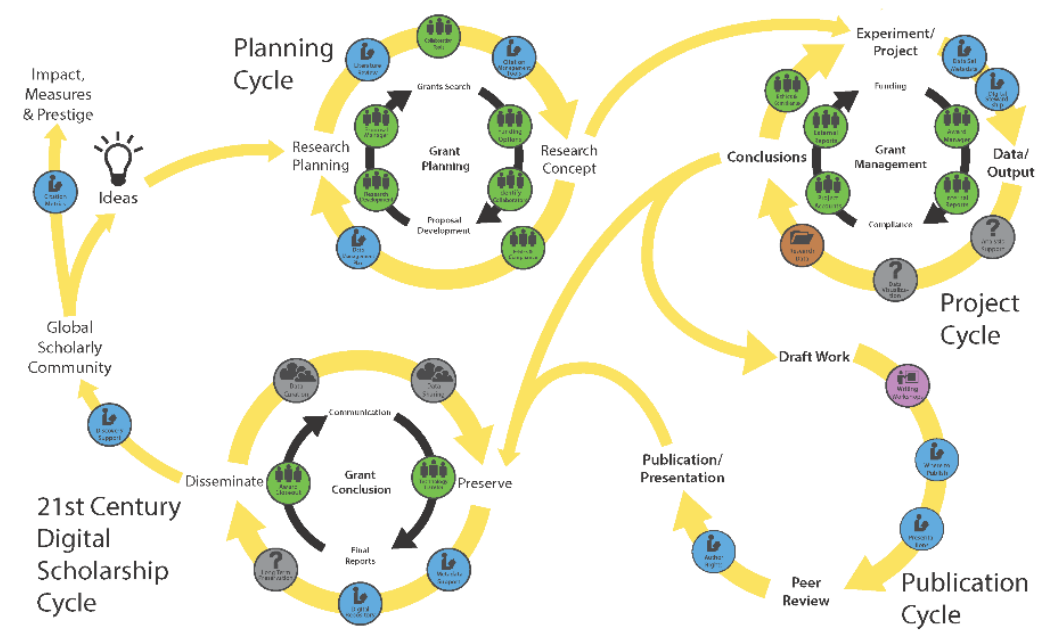

Figura 3. Ciclo de Vida de Pesquisa proposto pela UCF

No primeiro subciclo são descritas as atividades de planejamento da pesquisa, que podem incluir opções de financiamento, revisão da literatura, definição de questões relacionadas a aspectos éticos e de conformidade da pesquisa, planejamento da gestão de dados, identificação de possíveis colaboradores, entre outros. O segundo subciclo consiste na execução do planejamento desenvolvido na etapa anterior e trata de questões referentes ao próprio experimento da pesquisa, análise de dados, entre outros. Nesta etapa muitos dados de pesquisa ou dados científicos são criados. No terceiro subciclo o pesquisador deverá lidar com questões referentes à publicação dos relatos de pesquisa e, para isso, é necessário definir previamente o lugar onde deverá publicar, bem como definir questões relacionadas aos direitos de autoria. O quarto subciclo trata das questões de preservação e disseminação dos dados criados e tem como atividades centrais o compartilhamento e curadoria dos dados, estratégia de preservação a longo prazo, uso de repositórios digitais e suporte por meio de metadados. Por fim, o modelo proposto pela UCF apresenta ainda uma etapa que não é descrita como um subciclo. Aqui são consideradas questões relacionadas ao impacto da pesquisa e, nesse sentido, as métricas já desenvolvidas para avaliação e mensuração de impacto exercem papel fundamental. 
Este ciclo de vida de pesquisa possui algumas características apontadas por Cox e Tam (2018): é um modelo descritivo (busca descrever como o processo de pesquisa acontece na realidade), tem alto nível de abstração, é um modelo unidirecional (o fluxo de atividades segue em direção única) e as atividades são seriais (acontecem a partir de um conjunto de estágios). O ciclo de vida de pesquisa proposto pela UCF é um modelo consideravelmente complexo. Ele incorpora serviços institucionais de apoio à pesquisa em todo o processo, enfatizando também a conexão dos pesquisadores aos pontos de serviço incorporados no modelo proposto. Para Deng e Hu (2014), o ciclo de vida de pesquisa proposto pela UCF é construído no nível institucional com objetivos amplos: facilitar o planejamento estratégico da pesquisa, apresentar uma solução para todo o campus a partir das necessidades dos pesquisadores e conectar serviços de apoio à pesquisa oferecidos pela própria instituição e pesquisadores.

No tocante à Ciência Aberta, o ciclo de vida de pesquisa proposto pela UCF trata de acesso aberto, plataformas de suporte à pesquisa aberta, periódicos de acesso aberto e repositórios de acesso aberto. Este ciclo de vida de pesquisa ainda trata de questões inerentes à colaboração em pesquisa.

\section{Considerações Finais}

Os modelos de ciclo de vida de pesquisa foram os únicos encontrados na base de dados Scopus que fazem menção à Ciência Aberta. Todavia, analisando esses modelos propostos, é possível observar que ambos tratam de maneira muito limitada de questões específicas que compõem a Ciência Aberta. Convém ainda esclarecer que ambos os modelos desconsideram o fato de que a Ciência Aberta é multifacetada e engloba visões de mundo diferentes, conforme apontado por Fecher e Friesike (2014). Observa-se que o modelo de ciclo de vida de pesquisa proposto por Lyon (2016) aborda com profundidade a questão da transparência, como política e como aspecto fundamental para a reprodutibilidade da pesquisa. O artigo deixa a desejar, uma vez que não dá profundidade nas questões acerca das etapas do ciclo de vida de pesquisa proposto, ou seja, não são abordados os objetivos, as atividades e as tarefas prioritárias de cada fase.

Assim como o ciclo de vida de pesquisa proposto por Lyon (2016), Förstner et al. (2011) também propõem um ciclo de visa de pesquisa abordando questões muito específicas da Ciência Aberta. O modelo proposto tem como foco o dado de pesquisa e trata tangencialmente de tópicos como a colaboração e os Ambientes Virtuais de Pesquisa (VREs). Os ciclos de vida de pesquisa das universidades não são tão simplistas e acabam por englobar outros conceitos importantes no contexto da Ciência Aberta. O ciclo de vida de pesquisa da UCF é citado diversas vezes na literatura, uma vez que é bem representado graficamente e consegue tratar melhor da complexidade da Ciência Aberta, conforme aponta Picarra (2016).

Considerando o momento de transição vivido pela ciência, dado que esta tende a deixar seu modelo tradicional para migrar para um modelo embasado na Ciência Aberta, é de suma importância que os ciclos de vida de pesquisa sejam aderentes a este novo contexto. Espera-se que um modelo de ciclo de vida de pesquisa com foco na Ciência Aberta, além de que este trate com certa profundidade das fases que compõem o modelo, trate também de questões relacionadas aos atores envolvidos no processo de pesquisa, à abertura dos diferentes tipos de dados gerados nas diversas etapas do ciclo de vida de pesquisa, às ferramentas que podem auxiliar no processo de abertura da pesquisa, entre outros. Observa-se, portanto, que há aqui uma lacuna neste campo de 
pesquisa que necessita ser preenchida. Para que as pesquisas possam aderir aos conceitos da Ciência Aberta, é necessário que existam modelos de ciclo de vida de pesquisa que viabilizem este processo e englobem os pilares deste movimento, a Ciência Aberta.

\section{Agradecimentos}

O presente trabalho foi realizado com o apoio da Coordenação de Aperfeiçoamento de Pessoal de Nível Superior - BRASIL (CAPES) - Código de Financiamento 001.

\section{Referências}

Cox, A. M., \& Tam, W. W. T. (2018). A critical analysis of lifecycle models of the research process and research data management. Aslib Journal of Information Management.

Deng, S., \& Dotson, L. (2015). Redefining scholarly services in a research lifecycle. Creating research infrastructures in the 21st-century academic library: conceiving, funding, and building new facilities and staff, 77-92.

Deng, S., \& Hu, X. (2014). Creating a knowledge map for the research lifecycle.

Fecher, B., \& Friesike, S. (2014). Open science: one term, five schools of thought. In Opening science (pp. 17-47). Springer, Cham.

Fessari, R. (2015). Writing narrative style literature reviews. Medica Writing, 24(4).

Förstner, K., Hagedorn, G., Koltzenburg, C., Kubke, M. F., \& Mietchen, D. (2011). Collaborative platforms for streamlining workflows in Open Science. Nature Precedings, 1-1.

Funamori, M. (2017, July). Open Science and the Academy: A Theoretical Discussion. In 2017 6th IIAI International Congress on Advanced Applied Informatics (IIAIAAI) (pp. 109-115). IEEE.

Gessner, G. C., Eldermire, E., Tang, N., \& Tancheva, K. (2017). The Research Lifecycle and the Future of Research Libraries. In At the Helm: Leading Transformation: The Proceedings of the ACRL 2017 Conference (pp. 533-543).

Lyon, L. (2016). Transparency: the emerging third dimension of Open Science and Open Data. Liber quarterly, 25(4).

Picarra, M. (2016). Discussion Paper: Researchers and Open Science. Diponível em: < http://pasteur4oa.eu/sites/pasteur4oa/files/resource/Discussion\%20Paper_Researcher s\%20and\%20Open\%20Science.pdf $>$. Acesso em: 30 mar. 2020.

Ragon, B. (2019). Alignment of library services with the research lifecycle. Journal of the Medical Library Association: JMLA, 107(3), 384.

Toli, E., Sifacaki, E., Manola, N., Ioannidis, Y., Ross-Hellauer, T., Görögh, E., ... \& Woutersen-Windhouwer, S. (2018, August). Developing sustainable Open Science solutions in the frame of EU funded research: the OpenUP case. In Proceedings of the 14th International Symposium on Open Collaboration (pp. 1-10). 